\title{
AIP
}

\section{Quantum gates in hyperfine levels of ultracold alkali dimers by revisiting constrained-phase optimal control design}

A. Jaouadi, E. Barrez, Y. Justum, and M. Desouter-Lecomte

Citation: J. Chem. Phys. 139, 014310 (2013); doi: 10.1063/1.4812317

View online: http://dx.doi.org/10.1063/1.4812317

View Table of Contents: http://jcp.aip.org/resource/1/JCPSA6/v139/i1

Published by the AIP Publishing LLC.

\section{Additional information on J. Chem. Phys.}

Journal Homepage: http://jcp.aip.org/

Journal Information: http://jcp.aip.org/about/about_the_journal

Top downloads: http://jcp.aip.org/features/most_downloaded

Information for Authors: http://jcp.aip.org/authors

\section{ADVERTISEMENT}

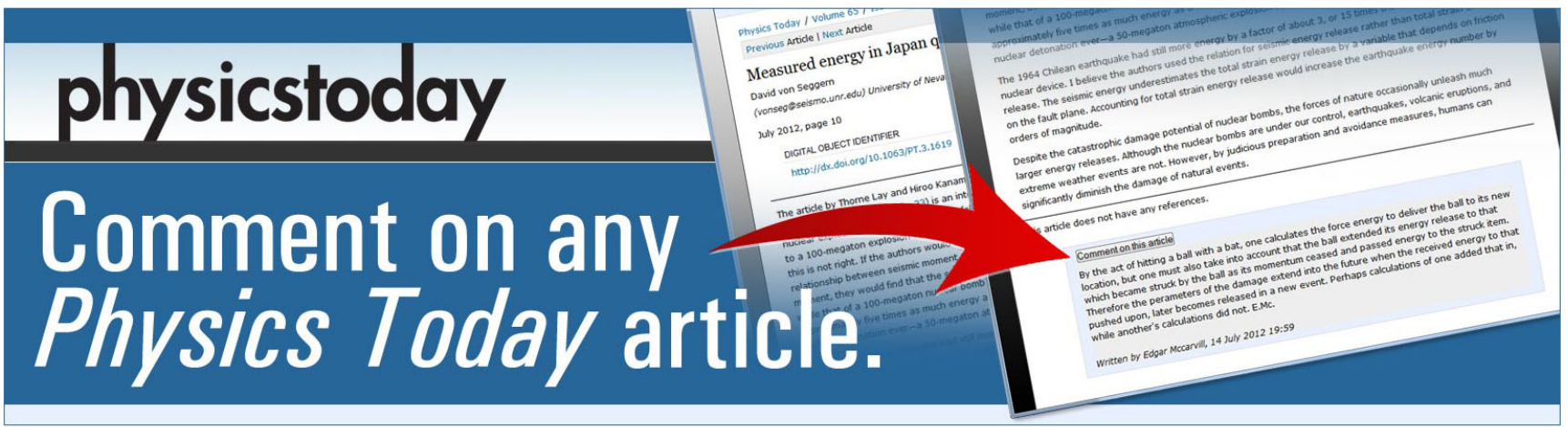




\title{
Quantum gates in hyperfine levels of ultracold alkali dimers by revisiting constrained-phase optimal control design
}

\author{
A. Jaouadi, ${ }^{1}$ E. Barrez, ${ }^{1}$ Y. Justum, ${ }^{1}$ and M. Desouter-Lecomte ${ }^{1,2, a)}$ \\ ${ }^{1}$ Laboratoire de Chimie Physique, UMR 8000 and CNRS, Université Paris-Sud, F-91405 Orsay, France \\ ${ }^{2}$ Département de Chimie, Université de Liège, Bât B6c, Sart Tilman, B4000 Liège, Belgium
}

(Received 15 November 2012; accepted 12 June 2013; published online 2 July 2013)

\begin{abstract}
We simulate the implementation of a 3-qubit quantum Fourier transform gate in the hyperfine levels of ultracold polar alkali dimers in their first two lowest rotational levels. The chosen dimer is ${ }^{41} \mathrm{~K}^{87} \mathrm{Rb}$ supposed to be trapped in an optical lattice. The hyperfine levels are split by a static magnetic field. The pulses operating in the microwave domain are obtained by optimal control theory. We revisit the problem of phase control in information processing. We compare the efficiency of two optimal fields. The first one is obtained from a functional based on the average of the transition probabilities for each computational basis state but constrained by a supplementary transformation to enforce phase alignment. The second is obtained from a functional constructed on the phase sensitive fidelity involving the sum of the transition amplitudes without any supplementary constrain. (C) 2013 AIP Publishing LLC. [http://dx.doi.org/10.1063/1.4812317]
\end{abstract}

\section{INTRODUCTION}

Information processing based on quantum mechanics exploiting superposed and entangled states is a promising way to speed up operations ${ }^{1,2}$ but it is expected that at least a hundred of qubits will be necessary to overcome the power of classical computers and no technology has reached this requirement nowadays. It remains not clear which material, photons, ${ }^{3}$ trapped ions, ${ }^{4,5}$ quantum dots, ${ }^{6}$ atoms, ${ }^{7}$ or polar molecules ${ }^{8,9}$ will be ultimately more efficient. ${ }^{10}$ Molecules offering long-lived nuclear spin states have been early investigated with nuclear magnetic resonance technology. ${ }^{11}$ Molecular rovibrational levels have also been proposed as promising candidates for encoding qubits in polyatomic or diatomic molecules. ${ }^{12-40}$ Ultracold polar molecules which can interact via dipole-dipole interaction are particularly interesting to create entanglement between neighboring molecules and to open the way toward qubit networks. ${ }^{41-48}$ Alkali dimers also possess a rich spin structure because both nuclei have a nonzero spin. The spin interactions and the coupling with the overall rotation lead to hyperfine levels which can be further manipulated in magnetic or electric fields. ${ }^{49,50}$ The strong state mixing resulting from the hyperfine interactions provide many possibilities for quantum logical operations. ${ }^{51,52}$ Our recent simulations by stimulated Raman adiabatic passage (STIRAP) or multi-target optimal control theory (MTOCT) have shown that high fidelity intramolecular or intermolecular quantum gates could be implemented in these hyperfine states of one or two neighboring molecules. ${ }^{53,54}$ However, in these previous works, we mainly focused on gates involving population inversion, for instance, in a quantum adder. In this paper, we consider a quantum Fourier transform (QFT) which requires realization of numerous phase gates. We simulate the implementation of a three-qubit gate in the hyperfine states

\footnotetext{
a) Author to whom correspondence should be addressed. Electronic mail: michele.desouter-lecomte@u-psud.fr
}

of the $N=0$ and $N=1$ rotational manifolds of the ${ }^{41} \mathrm{~K}^{87} \mathrm{Rb}$ dimer.

We take this example to revisit and illustrate the relation between the index chosen to measure the gate accuracy and that chosen to construct the functional in MTOCT. It seems intuitive to use the same quantity but it is not always the case in many previous applications. ${ }^{33,38,44,48,54,55}$ It has been discussed early that realizing a gate transformation by an optimal field requires a careful choice of the performance measure. ${ }^{56,57}$ The average transition probability for each computational basis state is not sufficient because the laser pulse is not able to drive any superposed state as it must do. On the contrary, the fidelity based on the sum of the transition amplitudes is a correct phase-sensitive measure. This fidelity criterion is used in many MTOCT works $32,34,38,39,44-46,48,53,54$ but with an optimal field derived from the probability functional by including a supplementary transition to enforce phase alignment. ${ }^{17,24}$ Here, we derive the optimal field using a functional based on the fidelity and we compare with the mixed counterintuitive procedure. A similar optimal field directly based on the fidelity has already been obtained by Krotov method. ${ }^{56}$

The outline of the paper is organized as follows: Sec. II summarizes theory and methods, i.e., the hyperfine Hamiltonian, the variational MTOCT, and the quantum Fourier transform. Results of the simulations are given in Sec. III where we compare the two strategies based on the different functionals and Sec. IV concludes.

\section{THEORY}

\section{A. Hyperfine levels}

The dipolar molecules are assumed to be trapped in a one-dimensional optical lattice whose frequency does not interfere with any internal excitation. We do not use the 
translational motion of the mass centers to realize the gates (see Ref. 58 for a recent OCT simulation using translational states of trapped atoms). As the molecules are ultracold, this translational motion corresponds to the ground state of the trapped well (a Gaussian function in the harmonic approximation). The mass centers are supposed to be fixed at their average position in each well.

We assume that the molecules are in the ground vibrational state of the ${ }^{1} \Sigma^{+}$ground electronic state. The internal Hamiltonian which includes interaction with a static magnetic field reads

$$
\hat{H}_{0}=\hat{H}_{r o t}+\hat{H}_{h f}+\hat{H}_{Z},
$$

$\hat{H}_{r o t}=B_{v=0} \mathbf{N}^{2}$ is the rotational Hamiltonian. The corresponding rotational constant is taken in Ref. 59. We focus here on the first rotational states of the ground vibrational state so that the vibration-rotation separation is plainly justified. The hyperfine structure in a ${ }^{1} \Sigma^{+}$electronic state involves three angular momenta: the rotational angular momentum $\mathbf{N}$ and the nuclear spins $\mathbf{I}_{1}$ and $\mathbf{I}_{2}$. The hyperfine Hamiltonian is discussed in Refs. 59 and 60, it reads

$$
\hat{H}_{h f}=\sum_{k=1}^{2} \mathbf{V}_{k} \cdot \mathbf{Q}_{k}+\sum_{k=1}^{2} c_{k} \mathbf{N} \cdot \mathbf{I}_{k}+c_{3} \mathbf{I}_{1} \cdot \mathbf{T} \cdot \mathbf{I}_{2}+c_{4} \mathbf{I}_{1} \cdot \mathbf{I}_{2},
$$

where the first contribution comes from the electric quadrupole $\mathbf{Q}_{k}$ coupling, the second one is the spin-rotation coupling with the nuclear spin $I_{k}$ with spin-rotation coupling constants $c_{1}$ and $c_{2}$. The third and fourth terms are the tensor and scalar interactions between the nuclear spins with spinspin coupling constants $c_{3}$ and $c_{4}$, respectively. The tensor $\mathbf{T}$ describes the angle dependence of the spin-spin coupling. For the considered alkali metal dimers, we have neglected the nuclear tensor spin-spin interaction because the constant $c_{3}$ is here an order of magnitude smaller than $c_{4}$. The values of the constants are taken from Refs. 59 and 60. The electric and magnetic fields are applied in the laboratory $Z$ direction. The Zeeman Hamiltonian for a magnetic field $\mathbf{B}$ is

$$
H_{Z}=-g_{r} \mu_{N} \mathbf{N} \cdot \mathbf{B}-\sum_{k=1}^{2} g_{k} \mu_{N} \mathbf{I}_{k} \cdot \mathbf{B}\left(1-\sigma_{k}\right)
$$

with $\mu_{N}$ is the nuclear magneton, $g_{r}$ is the rotational $g$ factor, $g_{k}$ is the nuclear $g$ factor of each nucleus $k$, and $\sigma_{k}$ is the nuclear shielding constant (isotropic part of the shielding tensor).

The hyperfine states are obtained by diagonalizing the Hamiltonian matrix in the basis set of uncoupled angular momentum functions $\left|N m_{N}\right\rangle\left|I_{1} m_{1}\right\rangle\left|I_{2} m_{2}\right\rangle \cdot{ }^{53,54}$ For a linearly polarized field, the electric dipole transition selection rules are $\Delta m_{F}=0, \Delta m_{1}=\Delta m_{2}=0$. We consider states with $m_{F}=0$ only. The eigenstates are denoted $|w\rangle$ $=\sum_{N m_{N} m_{1} m_{2}} c_{N m_{N} m_{1} m_{2}, w}\left|N m_{N} m_{1} m_{2}\right\rangle$ where $w$ labels the states by increasing energy. When there is no external field applied, the large number of allowed transitions due to the state mixing and the degeneracy would make the implementation and manipulation of the qubit states impractical. However, the Zeeman splitting reduces the number of allowed transi- tions. The Zeeman splitting in ${ }^{41} \mathrm{~K}^{87} \mathrm{Rb}$ is shown in Fig. 2 of Ref. 49.

The time dependent coupling with a microwave field $\varepsilon(t)$ is described at the electric dipolar approximation. For a pulse polarized along the laboratory $Z$ direction, the total Hamiltonian becomes

$$
\hat{H}(t)=\hat{H}_{0}-\hat{\mu}_{Z} \varepsilon(t)=\hat{H}_{0}-\hat{\mu}_{z} \cos (\theta) \varepsilon(t),
$$

where $\hat{\mu}_{Z}$ and $\hat{\mu}_{z}$ are the components of the dipole moment along the $Z$ axis in the laboratory frame and along the internuclear axis, respectively. Indeed, by using the tensorial notation, one has $\mu_{q}=\sum_{p}(-1)^{p} D_{q, p}^{(1)}(\theta, \phi, \chi) \mu_{p}$ where $q=0, \pm 1$ denotes the dipole components in the laboratory frame and $p=0, \pm 1$ designates the components in the molecular frame. The dipole for ${ }^{1} \Sigma^{+}$state being aligned along the $z$ internuclear axis, the summation over $p$ index reduces to the $p=0$ term, i.e., to the $\mu_{z}$ component. Knowing that $D_{0,0}^{(1)}(\theta, \phi, \chi)=\cos (\theta)$, one obtains relation (4).

\section{B. Optimal pulse design}

Designing a single pulse that drives a quantum gate can be done by the multi-target optimal control theory. ${ }^{13,18}$ The computational basis is defined by mapping the $2^{n}$ logical states of $n$ qubits on $2^{n}$ physical states $|k\rangle$ of the chosen system. The gate is represented by a $2^{n} \times 2^{n}$ matrix $O_{g}$ in the computational basis set

$$
O_{g}|k\rangle=\left|k_{f}\right\rangle \text {. }
$$

The output states $\left|k_{f}\right\rangle$ may be one of the computational basis state or a superposition of these states, for instance, in a HADAMARD gate $\left|0_{f}\right\rangle=(|0\rangle+|1\rangle) / \sqrt{2}$ and $\left|1_{f}\right\rangle$ $=(|0\rangle-|1\rangle) / \sqrt{2}$. The goal of the control is to find a universal pulse steering any basis state or any superposition toward the corresponding output at time $T$, i.e., $U(T, 0) \sum_{k} b_{k}|k\rangle$ $=\sum_{k} b_{k}\left|k_{f}\right\rangle$ where $U(T, 0)$ is the evolution operator with the time dependent Hamiltonian. Dynamics is carried out in a complete basis set of dimension larger than $2^{n}$ allowing transitory population of states orthogonal to the computational basis set. In this work, the performance of the laser pulse is measured by different criterions. The first one is the phase insensitive average transition probability of the $2^{n}$ inputs-outputs

$$
P=\sum_{k=1}^{2^{n}}\left|\left\langle k_{f} \mid \psi_{k}(T)\right\rangle\right|^{2} / 2^{n},
$$

where $\left|\psi_{k}(T)\right\rangle=U(T, 0)|k\rangle$ is the final state obtained with an initial condition in a computational basis state $\left|\psi_{k}(t=0)\right\rangle$ $=|k\rangle$ and $\left|k_{f}\right\rangle$ is the corresponding output of the gate [Eq. (5)]. The second criterion is the normalized fidelity which is phase sensitive $\mathrm{e}^{56,57}$

$$
F=\left|\operatorname{Tr}\left(O_{g}^{\dagger} U_{g}(T)\right)\right|^{2} / 2^{2 n}=\left|\sum_{k=1}^{2^{n}}\left\langle k_{f} \mid \psi_{k}(T)\right\rangle\right|^{2} / 2^{2 n},
$$

where $U_{g}(T)=P_{g} U(T) P_{g}$ and $P_{g}$ projects on the computational subspace. In the variational MTOCT, the field maximizes a functional constructed with a chosen objective under several constraints which limit the laser fluence and impose that the Schrödinger equation be satisfied at any 
time. Most of the MTOCT simulations have been carried out with an objective functional built from the average fidelity $P^{32,34,38,39,44-46,48,53,54}$ A phase constrain can be achieved by optimizing simultaneously a supplementary transformation involving a superposed state ${ }^{17,24}$

$$
|s\rangle=2^{-n / 2} \sum_{k=1}^{2^{n}}|k\rangle \rightarrow\left|s_{f}\right\rangle=2^{-n / 2} \sum_{k=1}^{2^{n}}\left|k_{f}\right\rangle e^{i \phi}
$$

where $\phi$ is a single phase taking any value between 0 and $2 \pi$. Then the usual probability functional reads ${ }^{61-63}$

$$
\begin{aligned}
J_{P}= & \sum_{k=1}^{2^{n}+1}\left|\left\langle k_{f} \mid \psi_{k}(T)\right\rangle\right|^{2}-\int_{0}^{T} \alpha(t) \varepsilon^{2}(t) d t \\
& -2 \Re e\left[\sum_{k=1}^{2^{n}+1}\left\langle k_{f} \mid \psi_{k}(T)\right\rangle \int_{0}^{T}\left\langle\lambda_{k}(t)\right| \partial_{t}\right. \\
& \left.+(i / \hbar)\left[H_{0}-\mu_{Z} \varepsilon(t)\right]\left|\psi_{k}(t)\right\rangle d t\right],
\end{aligned}
$$

where we assume a polarization along a $Z$ direction. The summation involves the $2^{n}$ transitions of the gate [Eq. (5)] and the supplementary constraint $|s\rangle \rightarrow\left|s_{f}\right\rangle$ [Eq. (8)]. The second term in the right member limits the laser energy via the penalty factor $\alpha(t)=\alpha_{0} / s(t)$ and $s(t)=\sin ^{2}(\pi t / T) . \lambda_{k}(t)$ is the Lagrange multiplier for the Schrödinger equation constraint. Variation of $\lambda_{k}$ and $\psi_{k}$ leads to evolution equations with initial and final conditions, respectively, ${ }^{64}$

$$
\begin{gathered}
\partial_{t}\left|\psi_{k}(t)\right\rangle=-(i / \hbar)\left[H_{0}-\mu_{Z} \varepsilon(t)\right]\left|\psi_{k}(t)\right\rangle \\
\left|\psi_{k}(t=0)\right\rangle=|k\rangle, \\
\partial_{t}\left|\lambda_{k}(t)\right\rangle=-(i / \hbar)\left[H_{0}-\mu_{Z} \varepsilon(t)\right]\left|\lambda_{k}(t)\right\rangle \\
\left|\lambda_{k}(t=T)\right\rangle=\left|k_{f}\right\rangle .
\end{gathered}
$$

The optimum field then takes the form

$$
\varepsilon_{P}(t)=-\frac{1}{\alpha(t)} \Im m\left[\sum_{k=1}^{2^{n}+1}\left\langle\lambda_{k}(t) \mid \psi_{k}(t)\right\rangle\left\langle\lambda_{k}(t)\left|\mu_{Z}\right| \psi_{k}(t)\right\rangle\right] .
$$

In this work, we compare this field with the field generated by the functional built from the phase sensitive fidelity $F$ without any supplementary transition

$$
\begin{aligned}
J_{F}= & \left|\sum_{k=1}^{2^{n}}\left\langle k_{f} \mid \psi_{k}(T)\right\rangle\right|^{2}-\int_{0}^{T} \alpha(t) \varepsilon^{2}(t) d t \\
& -2 \Re e\left[\sum_{j=1}^{2^{n}}\left\langle j_{f} \mid \psi_{j}(T)\right\rangle \sum_{k=1}^{2^{n}} \int_{0}^{T}\left\langle\lambda_{k}(t)\right| \partial_{t}\right. \\
& \left.+(i / \hbar)\left[H_{0}-\mu_{Z} \varepsilon(t)\right]\left|\psi_{k}(t)\right\rangle d t\right]
\end{aligned}
$$

where we have introduced a common factor containing the sum of the amplitudes $\left\langle j_{f} \mid \psi_{j}(T)\right\rangle$ for every $\lambda_{k}(t)$ term with the aim at decoupling the final conditions such as in Eq. (11). Details are given in the Appendix. Variation of $\lambda_{k}$ and $\psi_{k}$ leads to the same evolution equations [Eqs. (10) and (11)] with the optimal field

$$
\begin{aligned}
\varepsilon_{F}(t)= & -\frac{1}{\alpha(t)} \Im m\left[\sum_{j=1}^{2^{n}}\left\langle\lambda_{j}(t) \mid \psi_{j}(t)\right\rangle\right. \\
& \left.\times \sum_{k=1}^{2^{n}}\left\langle\lambda_{k}(t)\left|\mu_{Z}\right| \psi_{k}(t)\right\rangle\right] .
\end{aligned}
$$

A similar result has been obtained by Krotov's method. ${ }^{57}$ The MTOCT nonlinear equations are solved by the Rabitz iterative monotonous convergent algorithm. As suggested in Ref. 57, at each iteration step $i$ the field is obtained by $\varepsilon^{(i)}$ $=\varepsilon^{(i-1)}+\Delta \varepsilon^{(i)}$ where $\Delta \varepsilon^{(i)}$ is estimated by Eq. (12) or (14), respectively.

In the simulations presented below, we compare the two strategies using $\varepsilon_{P}(t)$ [Eq. (12)] computed by driving $2^{n}+1$ transitions and $\varepsilon_{F}(t)$ [Eq. (14)] involving $2^{n}$ transitions. The efficiency of the gate pulse is measured each time by the performance index $P$ and $F$. Further, we check the universality of the gate fields $\varepsilon_{P}(t)$ and $\varepsilon_{F}(t)$ on some arbitrary superposed states $\sum_{k} b_{k}|k\rangle$. We analyze the convergence of two state distances, of the fidelity. The state distances are the trace distance which is a measure for the distinguishability of two states $^{2,65,66}$

$$
d_{1}=\frac{1}{2} \operatorname{Tr} \sqrt{\left(\rho(T)-\rho_{t}\right)^{\dagger}\left(\rho(T)-\rho_{t}\right)}
$$

and the Hilbert-Schmidt distance ${ }^{67}$

$$
d_{2}=\sqrt{\operatorname{Tr}\left(\rho(T)-\rho_{t}\right)^{\dagger}\left(\rho(T)-\rho_{t}\right)}
$$

where $\rho(T)$ and $\rho_{t}$ are the density matrices of the final state $U(T, 0) \sum_{k} b_{k}|k\rangle$ and of the target state $\sum_{k} b_{k}\left|k_{f}\right\rangle$, respectively. We also estimate the state fidelity by the relation

$$
f=\sqrt{\operatorname{Tr}\left[\rho(T) \rho_{t}\right]} .
$$

Finally, for the converged gate pulse, an average gate fidelity is estimated. ${ }^{68,69}$ This average could be experimentally determined and can be obtained by choosing a set of quantum states forming an operator basis. A standard set of initial states can be built by the $4^{n}$ tensor-product states of the one-qubit states $|0\rangle,|1\rangle,(|0\rangle+|1\rangle) / \sqrt{2}$, and $(|0\rangle+i|1\rangle) / \sqrt{2}$. 2,70

\section{Quantum Fourier transform}

We consider a computational basis set $\left|2^{n}-1\right\rangle, \ldots$, $|0\rangle$ of a $n$-qubit register. Each logical state $|j\rangle=\mid j_{n-1}, \ldots$, $\left.j_{0}\right\rangle$ is defined by the value $j_{k}=0,1$ of each qubit $Q_{n}$, $\ldots, Q_{1} . j_{n-1}, \ldots, j_{0}$ gives the binary representation of $j$. The quantum Fourier transform modifies a generic $n$-qubit state $\sum_{j=0}^{2^{n}-1} a_{j}|j\rangle$ into a state $\sum_{j=0}^{2^{n}-1} \bar{a}_{j}|j\rangle$ so that the vector $\left(\bar{a}_{0}, \ldots \bar{a}_{2^{n}-1}\right)$ is the discrete Fourier transform of the vector $\left(a_{0}, \ldots, a_{2^{n}-1}\right)$. Each component of the output vector is thus given by $\bar{a}_{l}=2^{-n / 2} \sum_{j=0}^{2^{n}-1} e^{2 \pi i j l / 2^{n}} a_{j}$. The global unitary 


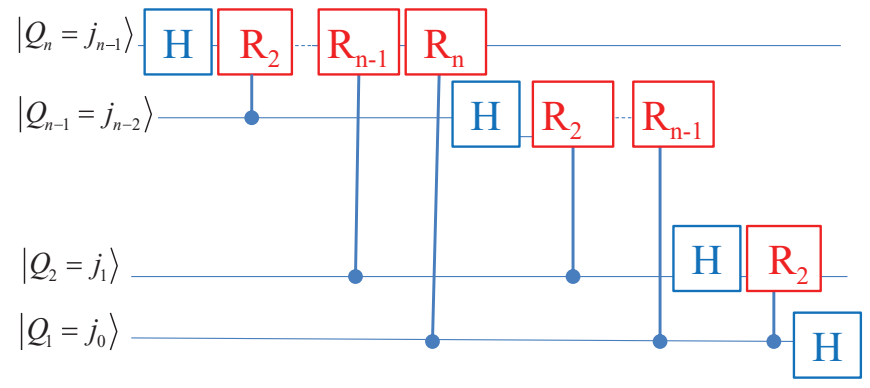

FIG. 1. Circuit implementing the quantum Fourier transform with $n$ qubits $Q_{n} \ldots Q_{1} . \mathrm{H}$ is the Hadamard gate and $\mathrm{R}_{k}$ is the controlled phase shift of $\exp (i \phi)$ with $\phi=2 \pi / 2^{k}$. The control qubit is indicated by a full circle. The SWAP gates which reverse the order of the qubits at the end of the circuit are not drawn.

transformation is

$$
\mathrm{QFT}_{n}=2^{-n / 2}\left(\begin{array}{ccccc}
1 & 1 & 1 & \cdots & 1 \\
1 & \omega & \omega^{2} & & \omega^{2^{n}-1} \\
1 & \omega^{2} & \omega^{4} & & \omega^{2\left(2^{n}-1\right)} \\
\vdots & & & & \\
1 & \omega^{2^{n}-1} & \omega^{2\left(2^{n}-1\right)} & & \omega^{\left(2^{n}-1\right)\left(2^{n}-1\right)}
\end{array}\right),
$$

where $\omega=\exp \left(2 i \pi / 2^{n}\right)$. It could be decomposed into a circuit of elementary gates involving $n$ HADAMARD gates and $n(n-1) / 2$ controlled phase gates as shown in Fig. 1. The HADAMARD gate transformed each qubit state in a superposition as follows: $|0\rangle \rightarrow(|0\rangle+|1\rangle) / \sqrt{2}$ and $|1\rangle$ $\rightarrow(|0\rangle-|1\rangle) / \sqrt{2}$. The phase gate $\mathrm{R}_{k}$ adds a phase $\exp (i \phi)$ with $\phi=2 \pi / 2^{k}$ to state $|1\rangle$ only, i.e., $|0\rangle \rightarrow|0\rangle$ and $|1\rangle$ $\rightarrow e^{i \phi}|1\rangle$. The controlled- $\mathrm{R}_{k}$ phase gate does this phase shift in the target qubit only if the control qubit is in state $|1\rangle$ so that $|00\rangle \rightarrow|00\rangle,|01\rangle \rightarrow|01\rangle,|10\rangle \rightarrow|10\rangle$, and $|11\rangle \rightarrow e^{i \phi}|11\rangle$. At the end, $n / 2$ or $(n-1) / 2$ SWAP operations $Q_{n} \rightarrow Q_{1}$, $Q_{n-1} \rightarrow Q_{2} \ldots$ (not shown in Fig. 1) are used to reorder the qubits in the output $\left(Q_{n}, Q_{n-1} \ldots Q_{2}, Q_{1} \rightarrow Q_{1}\right.$, $\left.Q_{2} \ldots Q_{n-1}, Q_{n}\right)$.

\section{RESULTS}

The dimer is ${ }^{41} \mathrm{~K}^{87} \mathrm{Rb}\left(I_{1}=I_{2}=3 / 2\right)$ and the magnetic field is $500 \mathrm{G}$. The eight computational states of the three qubits $Q_{3} Q_{2} Q_{1}$ are mapped onto eight states belonging to the manifolds $N=0$ and $N=1$. The four states $|000\rangle,|001\rangle$, $|010\rangle$, and $|011\rangle$ are encoded in states $|4\rangle,|7\rangle,|10\rangle$, and $|13\rangle$ with $N=0$. The states $|100\rangle,|101\rangle,|110\rangle$, and $|111\rangle$ are encoded in $|30\rangle,|36\rangle,|41\rangle$, and $|47\rangle$ with $N=1$. Table I gives the non-vanishing dipole matrix elements between the states with $N=0$ and $N=1$. The dominant uncoupled states in the eigenvectors are also given.

All the simulations are carried out by integrating the coupled equations by the 4 th order Runge Kutta method ${ }^{71}$ with a time step of $48.5 \mathrm{ps}$. The trial field is a sum of 16 sine square pulses $\varepsilon_{0}(t)=A_{0} \sin ^{2}(\pi t / T) \cos (\omega t)$ with the carrier frequencies connecting the four selected states in $N=0$ to the four ones in $N=1$ (see Table I). The maximum of the envelope $A_{0}$ is the same for each pulse.

The physical implementation of a QFT by the circuit split into seven elementary gates $H^{(3)} R_{2}^{(3)} R_{3}^{(3)} H^{(2)} R_{2}^{(2)} H^{(1)} S W$ should increase the total pulse duration and lead to an accumulation of physical imprecision and decoherence. MTOCT can in principle optimize a circuit in one single step. ${ }^{28,31,35,36,58}$ To ensure small gate error below experimental error correction threshold, the fidelity must reach at least 0.99999. We first choose the pulse duration $T$ and the amplitude $A_{0}$ of the trial field. For each case and for each functional, we determine the best penalty factor $\alpha_{0}$ leading to the fastest convergence. However, the main criterion to select $T$ and $A_{0}$ is the shape of the pulse which must be experimentally feasible and robust. So we analyze the Fourier transform in the frequency domain to disregard conditions for which the spectrum presents a large background requiring filtration procedures. A good compromise for the time duration appears to be $108.5 \mu \mathrm{s}\left(4.5 \times 10^{12}\right.$ a.u. $)$. Table II gives the chosen penalty factor and the number of iterations leading to a fidelity equal to 0.99999 for the two fields $\varepsilon_{P}(t)$ and $\varepsilon_{F}(t)$ with different amplitudes $A_{0}$. The order of magnitude of the fields is fixed by $A_{0}$. At any iteration, the corrections $\Delta \varepsilon^{(i)}$ are given by

TABLE I. Non-vanishing dipole matrix elements in a.u. for linear polarization between the $N=0$ and the $N=1$ manifolds for ${ }^{41} \mathrm{~K}^{87} \mathrm{Rb}\left(I_{1}=I_{2}=3 / 2\right)$ in a magnetic field $500 \mathrm{G}$. The main uncoupled component $\left|N m_{N} m_{1} m_{2}\right\rangle$ and the corresponding weight $c_{N m_{N} m_{1} m_{2}, w}^{2}$ of the $|w\rangle$ eigenvectors are specified. The eight computational states chosen to encode the 3-qubit states are in bold.

\begin{tabular}{|c|c|c|c|c|c|c|}
\hline$|w\rangle$ & Main component & Weight & $\mid \begin{array}{c}\mathbf{4} \\
|0,0,-3 / 2,3 / 2\rangle \\
1\end{array}$ & $|0,0,-1 / 2,1 / 2\rangle$ & $\mid 0, \begin{array}{c}\mathbf{1 0} \\
1\end{array}$ & $|0,0,3 / 2,-3 / 2\rangle \mid$ \\
\hline 19 & $|1,0,-3 / 2,3 / 2\rangle$ & 0.90 & $-1.22 \times 10^{-1}$ & $5.81 \times 10^{-3}$ & $-4.14 \times 10^{-5}$ & $-2.24 \times 10^{-5}$ \\
\hline 24 & $|1,-1,-1 / 2,3 / 2\rangle$ & 0.88 & $2.88 \times 10^{-2}$ & $2.47 \times 10^{-2}$ & $-6.27 \times 10^{-5}$ & $-1.03 \times 10^{-4}$ \\
\hline 30 & $|1,-1,1 / 2,1 / 2\rangle$ & 0.92 & $-7.96 \times 10^{-3}$ & $-2.25 \times 10^{-3}$ & $3.89 \times 10^{-3}$ & $6.71 \times 10^{-4}$ \\
\hline 36 & $|1,1,-3 / 2,1 / 2\rangle$ & 0.88 & $-2.59 \times 10^{-2}$ & $-2.38 \times 10^{-2}$ & $-1.32 \times 10^{-3}$ & $-2.67 \times 10^{-4}$ \\
\hline 40 & $|1,0,-1 / 2,1 / 2\rangle$ & 0.92 & $-5.06 \times 10^{-3}$ & $1.23 \times 10^{-1}$ & $2.30 \times 10^{-5}$ & $6.04 \times 10^{-4}$ \\
\hline 41 & $|1,-1,3 / 2,-1 / 2\rangle$ & 0.86 & $-2.87 \times 10^{-4}$ & $5.24 \times 10^{-3}$ & $-1.40 \times 10^{-2}$ & $-3.48 \times 10^{-2}$ \\
\hline 47 & $|1,1,-1 / 2,-1 / 2\rangle$ & 0.89 & $-7.77 \times 10^{-4}$ & $9.77 \times 10^{-3}$ & $9.43 \times 10^{-3}$ & $1.19 \times 10^{-2}$ \\
\hline 50 & $|1,0,1 / 2,-1 / 2\rangle$ & 0.91 & $3.97 \times 10^{-5}$ & $3.53 \times 10^{-4}$ & $-1.23 \times 10^{-1}$ & $5.07 \times 10^{-3}$ \\
\hline 53 & $|1,0,3 / 2,-3 / 2|$ & 0.91 & $1.21 \times 10^{-7}$ & $9.98 \times 10^{-5}$ & $-3.43 \times 10^{-3}$ & $1.22 \times 10^{-1}$ \\
\hline 61 & $|1,1,1 / 2,-3 / 2\rangle$ & 0.90 & $5.57 \times 10^{-5}$ & $1.87 \times 10^{-5}$ & $3.39 \times 10^{-2}$ & $1.30 \times 10^{-2}$ \\
\hline
\end{tabular}


TABLE II. Convergence of the fidelity up to 0.99999 obtained by the two fields $\varepsilon_{P}(t)$ [Eq. (12)] and $\varepsilon_{F}(t)$ [Eq. (14)] for different amplitudes $A_{0}$ of the trial pulses and the best penalty factor.

\begin{tabular}{lccccc}
\hline & \multicolumn{2}{c}{$\varepsilon_{F}(t)$} & & \multicolumn{2}{c}{$\varepsilon_{P}(t)$} \\
\cline { 2 - 4 }$A_{0}\left(\mathrm{~V} \mathrm{~cm}^{-1}\right)$ & $\alpha_{0}$ & Iteration & & $\alpha_{0}$ & Iteration \\
\hline 51.42 & $10^{8}$ & 2140 & & $1.5 \times 10^{7}$ & 2500 \\
5.14 & $10^{9}$ & 320 & & $2 \times 10^{8}$ & 740 \\
1.03 & $5 \times 10^{8}$ & 550 & & $2 \times 10^{8}$ & 710 \\
\hline \hline
\end{tabular}

Eq. (12) or (14) which contain a quite different factor multiplying the dipole matrix element so that the best convergence is reached for different penalty factors. It is always smaller for $J_{P}$ by about one order of magnitude in the present case. For a given guess field, one observes that, for the optimal penalty factor, $J_{F}$ always converges the first.

As discussed below, according to the Fourier transform criterion, we select the trial field amplitude $A_{0}=5.14 \mathrm{~V} \mathrm{~cm}^{-1}$ $\left(10^{-9}\right.$ a.u.). Figure 2 shows the convergence of the fidelity $F$ [Eq. (7)] for different $\alpha_{0}$ obtained with the fields $\varepsilon_{F}(t)$ (panel (a)) and $\varepsilon_{P}(t)$ (panel (b)), respectively, for $T=108.5 \mu \mathrm{s}$. The optimal $\alpha_{0}$ (red line in Figure 2) is $10^{9}$ for $J_{F}$ and $2 \times 10^{8}$ for $J_{P}$. By using the best $\alpha_{0}, \varepsilon_{F}(t)$ makes the fidelity converge up to 0.99999 faster than $\varepsilon_{P}(t)$. Comparing the convergence for the same value of $\alpha_{0}$ would lead to the bad conclusion that $J_{P}$ converges the first. For instance, for $\alpha_{0}=2 \times 10^{8}$ both curves cross after 245 iterations for $F=0.965$.

Figure 3 compares the convergence of the performance measured by the average probability $P$ and by the fidelity $F$ for the best penalty factor in each strategy $\left(10^{9}\right.$ for $J_{F}$ and 2 $\times 10^{8}$ for $J_{P}$ ) in the chosen conditions $T=108.5 \mu \mathrm{s}$ and $A_{0}$ $=5.14 \mathrm{~V} \mathrm{~cm}^{-1}$. Both index are very close with $J_{F}$ but, as expected, $F$ converges slower than $P$ with $J_{P}$ since this functional is not directly built from $F$.

Figure 4 shows the optimal fields $\varepsilon_{F}(t)$ (panel (a)) and $\varepsilon_{P}(t)$ (panel (b)) and the square modulus of their Fourier transforms for the iteration number giving a fidelity of 0.99999 in the selected conditions of Figure $3(T=108.5 \mu \mathrm{s}$ and

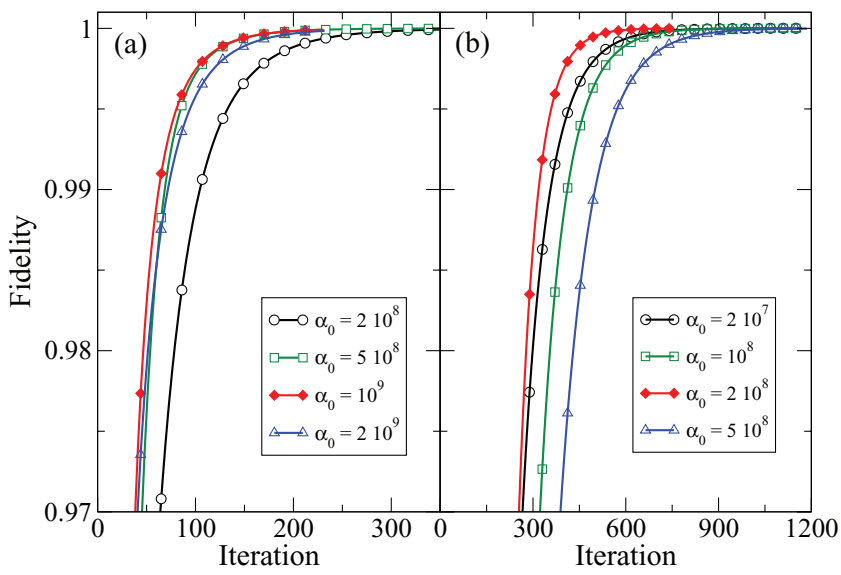

FIG. 2. Fidelity $F$ of the 3-qubit QFT driven by the optimal field with a pulse duration $108.5 \mu \mathrm{s}$ and an initial amplitude $A_{0}=5.14 \mathrm{~V} \mathrm{~cm}^{-1}$ for different penalty factors $\alpha_{0}$. Panel (a) $J_{F}$ [Eq. (13)], panel (b) $J_{P}$ [Eq. (9)].

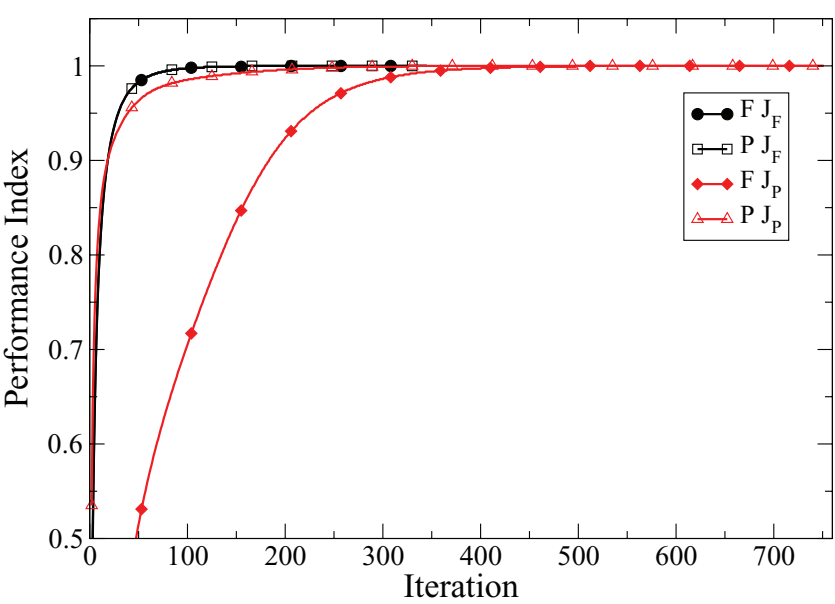

FIG. 3. Convergence of the fidelity $F$ and the average probability $P$ obtained with the best penalty factor in the two functionals $J_{F}\left(\alpha_{0}=10^{9}\right)$ or $J_{P}\left(\alpha_{0}\right.$ $=2 \times 10^{8}$ ) for the 3-qubit QFT. $T=108.5 \mu$ s and $A_{0}=5.14 \mathrm{~V} \mathrm{~cm}^{-1}$.

$\left.A_{0}=5.14 \mathrm{~V} \mathrm{~cm}^{-1}\right)$. The optimized fields and even their complicated shapes still have a Fourier transform spectrum very close to that of the trial pulse. The peaks correspond to the transition frequencies between the states $4,7,10$, and 13 belonging to the $N=0$ manifold to states $30,36,41$, and 47 of the $N=1$ manifold (see Table I). Both fields are very similar as can be also seen in Figure 5 where are displayed the spectrograms obtained by a Gabor transform ${ }^{72}$ of the optimal spectra. One only observes a weak variation of intensity for some peaks. The initial amplitude $A_{0}$ determines the initial variation of the field intensity. With the present choice $A_{0}$ $=5.14 \mathrm{~V} \mathrm{~cm}^{-1}$, the increase of the total energy $\int_{0}^{T} \varepsilon_{F / P}^{2}(t) d t$ is only $0.8 \%$ (for $\varepsilon_{F}$ ) and $2.4 \%$ (for $\varepsilon_{P}$ ). When the variation of the total energy is weak, the optimal spectrum remains simple without unrealistic background. A counter example is given below.

In Figure 6, we illustrate the universality of the gate pulses of Figure 4 by driving the QFT gate on an arbitrary
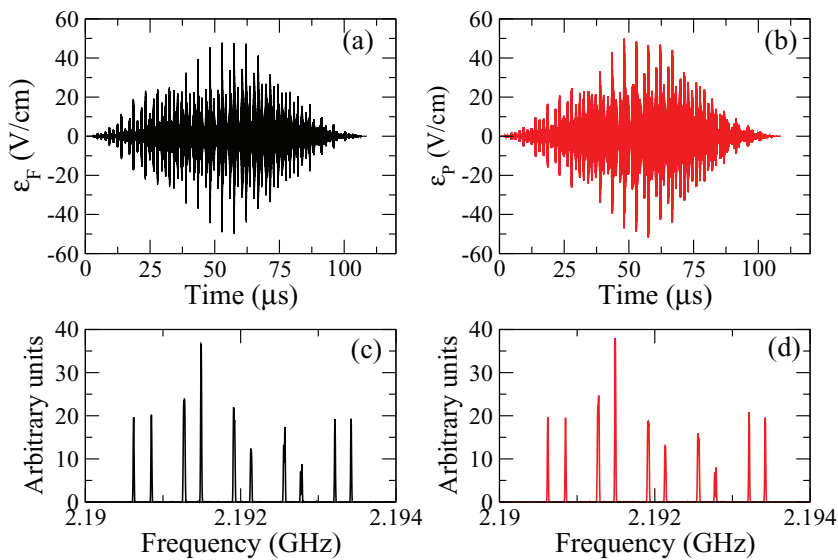

FIG. 4. Optimal field driving the 3-qubit QFT with a fidelity of 0.99999 in one step and the square modulus of their Fourier transform. Panel (a): $\varepsilon_{F}(t)$ (320 iterations, $\alpha_{0}=10^{9}$ ), panel (b): $\varepsilon_{P}(t)$ (740 iterations, $\alpha_{0}=2 \times 10^{8}$. The 16 intense peaks correspond to those of the guess field. $T=108.5 \mu \mathrm{s}$ and $A_{0}=5.14 \mathrm{~V} \mathrm{~cm}^{-1}$. 


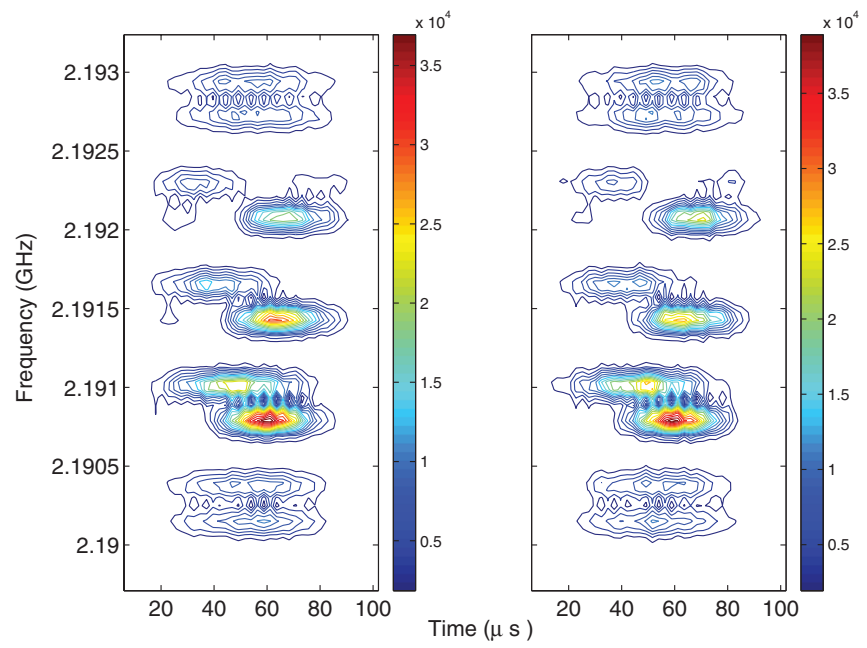

FIG. 5. Spectrograms of the control pulses presented in Figure 4. (Left panel) $\varepsilon_{F}(t)$, (right panel) $\varepsilon_{P}(t)$. The intensity scale, in arbitrary units, is the same for both cases.

initial superposed state

$$
\begin{aligned}
|\psi\rangle= & \frac{1}{2 \sqrt{2}}(|0,0,0\rangle-|0,0,1\rangle+|0,1,0\rangle-|0,1,1\rangle \\
& +|1,0,0\rangle-|1,0,1\rangle+|1,1,0\rangle-|1,1,1\rangle) \\
\rightarrow & \left|\psi_{f}\right\rangle=|1,0,0\rangle .
\end{aligned}
$$

The evolution of the distances $d_{1}$ [Eq. (15)] and $d_{2}$ [Eq. (16)] between the evolving state and the target as a function of the iteration number are drawn in panel (a) and the fidelity [Eq. (17)] in panel (b). The very small values of the final distances confirm the quality of the field in each case. The distances converge more quickly with $\varepsilon_{F}(t)$ when the optimal penalty factor is used.

To go beyond this single test on an arbitrary given state, we compute the average gate fidelity and the worst case (minimum value) over the $2^{3} \times 2^{3}$ initial states which are the tensor- products of a standard $2^{2}$ basis set for the one-qubit case: $|0\rangle,|1\rangle,(|0\rangle+|1\rangle) / \sqrt{2}$, and $(|0\rangle+i|1\rangle) / \sqrt{2}$., ${ }^{2,70}$ The
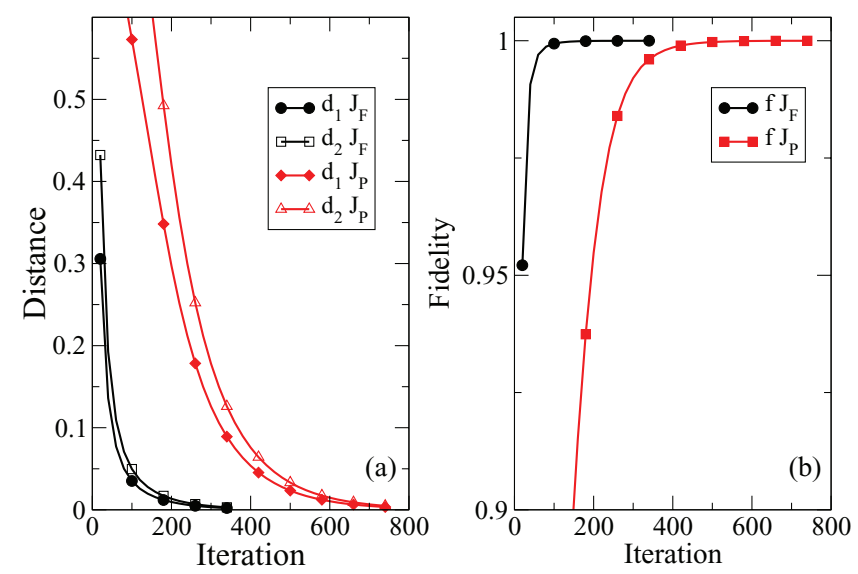

FIG. 6. Convergence of the trace distance $d_{1}$ and the Hilbert Schmidt distance $d_{2}$ (panel (a)) and of the fidelity (panel (b)) for the 3-qubit QFT acting on the superposed state $|\psi\rangle$ [Eq. (19)] driven by the optimal field obtained from the functionals $J_{F}\left(\alpha=10^{9}\right)$ and $J_{P}\left(\alpha=2 \times 10^{8}\right) . T=108.5 \mu \mathrm{s}$ and $A_{0}=5.14 \mathrm{~V} \mathrm{~cm}^{-1}$.
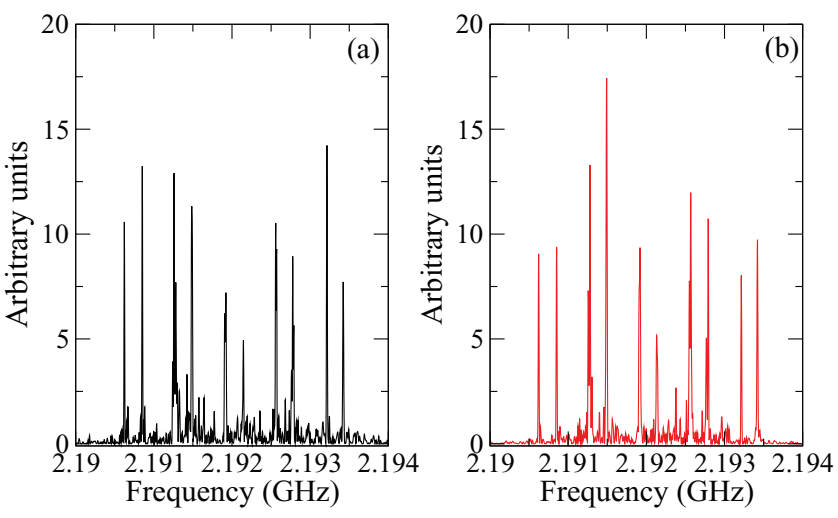

FIG. 7. Square modulus of the Fourier transform of the gate field driving the 3-qubit QFT with a fidelity of 0.99999. Panel (a): $\varepsilon_{F}(t)$ (550 iterations), panel (b): $\varepsilon_{P}(t)$ (707 iterations). $T=108.5 \mu \mathrm{s}$ and $A_{0}=1.03 \mathrm{~V} \mathrm{~cm}^{-1}$. The optimal $\alpha_{0}$ are given in Table II.

gate pulse $\varepsilon_{F}(t)$ lead to an average fidelity of 0.9999974 and a worst case of 0.99999295 while $\varepsilon_{P}(t)$ gives 0.9999704 and 0.99998651 , respectively.

Finally, we briefly discuss why we discard the low initial amplitude $1.03 \mathrm{~V} \mathrm{~cm}^{-1}\left(2 \times 10^{-10}\right.$ a.u. $)$ even if the convergence is easy (see Table II). Figure 7 shows the corresponding spectra for the optimal $\alpha_{0}$. For each strategy, the spectrum is contaminated by a large background of unrealistic frequencies which would require filtration and new iterations with the filtered field. The initial energy of the trial field is too low and gives rise to an important increase of the total energy pulse which is doubled during the first hundred iterations. This illustrates that a fast convergence is not a sufficient criterion to get a simple spectrum. The choice of the initial energy of guess field is also important to avoid a rapid variation of the energy during the optimization.

\section{CONCLUDING REMARKS}

Two points are illustrated in this work. First, we simulate the implementation of gates involving controlled-phase transformations and this completes our previous simulations ${ }^{53,54}$ in the hyperfine structure of cold alkali dimers which mainly involved population inversions. Second, we discuss the phase alignment in MTOCT.

(i) This example confirms that very high fidelity logical operations can be implemented in the hyperfine structure the splitting of which being modulated by a magnetic field. One of the achievements of this work is to optimize the QFT involving a lot of phase gates. The onestep QFT gate has been optimized with an average gate fidelity larger than 0.99999 in about $100 \mu \mathrm{s}$. A single gate such as the first Hadamard in Figure 1 requires at least $40 \mu$ s with a similar guess field. The pulse frequencies belong to the microwave domain for which shaping technologies are available. To get a simple spectrum without unrealistic background, the trial field must be carefully chosen in order to avoid a large variation of the total energy during the first iterations. It is obvious that further work remain to be done to manipulate registers of 
several dozens of qubits. However, realizing intramolecular gates is a first step and the dipole-dipole interaction could be used to drive intramolecular gates. ${ }^{41,44-46,54}$ The coupling with the translational motion in the trap could also be an interesting perspective.

(ii) It is well known that two strategies exist to enforce a good phase alignment with variational OCT. The $J_{F}$ Kosloff method ${ }^{57}$ involves the $2^{n}$ transitions of the unitary transformation only while the strategy proposed by de Vivie-Riedle ${ }^{18,19}$ uses a functional $J_{P}$ built with the average probability with $2^{n}+1$ transitions. We complete the previous discussions where $J_{F}$ was compared with $J_{P}$ without the inclusion of the phase constraint. ${ }^{57}$ As the expressions of the corresponding fields are different, it is not relevant to compare their behavior for the same penalty factor since the average pulse energy is then different for similar pulse duration and guess field. We have thus compared the convergence for the best penalty factor in each strategy and obviously the $J_{F}$ is then more efficient as it could be expected since it is directly based on a phase sensitive index.

\section{ACKNOWLEDGMENTS}

We wish to thank Dr. P. Pellegrini for the code Hfs.f90 computing the hyperfine levels. We also thank Dr. O. Atabek and Dr. M. Ndong for helpful discussions and interesting comments. This work was supported by Triangle de la Physique under Contract No. 2011-040T O.A.

\section{APPENDIX: OPTIMAL FIELD FROM THE FIDELITY FUNCTIONAL}

In the variational MTOCT, one first constructs an objective functional to be maximized under several constraints. We want to derive the expression of the optimal field when the objective is the phase sensitive factor $F$ $=\left|\sum_{k=1}^{2^{n}}\left\langle k_{f} \mid \psi_{k}(T)\right\rangle\right|^{2} / 2^{2 n}$. We make use of a functional similar to that proposed in the early work of Zhu, Botina, and Rabitz $^{61}$ in order to maximize a transition probability and generalized to the multi-target case ${ }^{13}$

$$
\begin{aligned}
J_{F}= & \left|\sum_{k=1}^{2^{n}}\left\langle k_{f} \mid \psi_{k}(T)\right\rangle\right|^{2}-\int_{0}^{T} \alpha(t) \varepsilon^{2}(t) d t \\
& -2 \Re e\left[\sum_{j=1}^{2^{n}}\left\langle j_{f} \mid \psi_{j}(T)\right\rangle \sum_{k=1}^{2^{n}} \int_{0}^{T}\left\langle\lambda_{k}(t)\right| \partial_{t}\right. \\
& \left.+(i / \hbar)\left[H_{0}-\mu_{Z} \varepsilon(t)\right]\left|\psi_{k}(t)\right\rangle d t\right] .
\end{aligned}
$$

The second term with penalty factor $\alpha(t)$ constraints the laser energy and the third one imposes the Schrödinger equation to be verified at all times. The corresponding Lagrange multipliers are $\lambda_{k}(t)$. Here, each term $k$ of the summation over the $N$ transitions is multiplied by the same sum $\sum_{j=1}^{2^{n}}\left\langle j_{f} \mid \psi_{j}(T)\right\rangle$ which is time invariant. ${ }^{61}$ This trick will lead to final conditions for the propagation of $\lambda_{k}(t)$ which are simply the target states. One has then to apply the variation of the variables $\psi_{k}(t), \lambda_{k}(t), \varepsilon(t)$, and impose $\delta J=0$. Variation of the $\lambda_{k}(t)$ gives

$$
\begin{aligned}
\delta_{\lambda_{l}} J_{F}= & -2 \Re e \sum_{j=1}^{2^{n}}\left\langle j_{f} \mid \psi_{j}(T)\right\rangle \int_{0}^{T}\left\langle\delta \lambda_{l}(t)\right| \partial_{t} \\
& +(i / \hbar)\left[H_{0}-\mu_{Z} \varepsilon(t)\right]\left|\psi_{l}(t)\right\rangle d t .
\end{aligned}
$$

$\delta \lambda_{l}$ being arbitrary, this means that the function $\psi_{l}(t)$ must satisfy the time-dependent Schrödinger equation

$$
\begin{gathered}
\partial_{t}\left|\psi_{l}(t)\right\rangle=-(i / \hbar)\left[H_{0}-\mu_{Z} \varepsilon(t)\right]\left|\psi_{l}(t)\right\rangle \text { with } \\
\left|\psi_{l}(t=0)\right\rangle=|l\rangle .
\end{gathered}
$$

Next, variation of the $\psi_{k}(t)$ leads to

$$
\begin{aligned}
\delta_{\psi_{l}} J_{F}= & 2 \Re e\left(\sum_{j=1}^{2^{n}}\left\langle j_{f} \mid \psi_{j}(T)\right\rangle\left\langle l_{f} \mid \delta \psi_{l}(T)\right\rangle\right) \\
& -2 \Re e\left(\left\langle l_{f} \mid \delta \psi_{l}(T)\right\rangle \sum_{k=1}^{2^{n}} \int_{0}^{T}\left\langle\lambda_{k}(t)\right| \partial_{t}\right. \\
& \left.+(i / \hbar)\left[H_{0}-\mu_{Z} \varepsilon(t)\right]\left|\psi_{k}(t)\right\rangle d t\right) \\
& -2 \Re e\left[\sum_{j=1}^{2^{n}}\left\langle j_{f} \mid \psi_{j}(T)\right\rangle \int_{0}^{T}\left\langle\lambda_{l}(t)\right| \partial_{t}\right. \\
& \left.+(i / \hbar)\left[H_{0}-\mu_{Z} \varepsilon(t)\right]\left|\delta \psi_{l}(t)\right\rangle d t\right]
\end{aligned}
$$

$\delta \psi_{l}$ being arbitrary, it may be chosen time-independent $\delta \psi_{l}(t)$ $=\delta \psi_{l}$. Then Eq. (A4) becomes

$$
\begin{aligned}
\delta_{\psi_{l}} J_{F}= & 2 \Re e\left(\sum_{j=1}^{2^{n}}\left\langle j_{f} \mid \psi_{j}(T)\right\rangle\left\langle l_{f} \mid \delta \psi_{l}\right\rangle\right) \\
& -2 \Re e\left[\sum_{j=1}^{2^{n}}\left\langle j_{f} \mid \psi_{j}(T)\right\rangle \int_{0}^{T}\left\langle\lambda_{l}(t)\right|\right. \\
& \left.\times(i / \hbar)\left[H_{0}-\mu_{Z} \varepsilon(t)\right]\left|\delta \psi_{l}\right\rangle d t\right]
\end{aligned}
$$

or by using the adjoint operator

$$
\begin{aligned}
\delta_{\psi_{l}} J_{F}= & 2 \Re e\left(\sum_{j=1}^{2^{n}}\left\langle j_{f} \mid \psi_{j}(T)\right\rangle\left\langle l_{f} \mid \delta \psi_{l}\right\rangle\right) \\
& -2 \Re e\left[\sum_{j=1}^{2^{n}}\left\langle j_{f} \mid \psi_{j}(T)\right\rangle\right. \\
& \left.\times \int_{0}^{T}\left\langle(-i / \hbar)\left[H_{0}-\mu_{Z} \varepsilon(t)\right] \lambda_{l}(t) \mid \delta \psi_{l}\right\rangle d t\right] .
\end{aligned}
$$

$\delta_{\psi_{l}} J_{F}=0$ if $\lambda_{l}(t)$ satisfies the Schrödinger equation with final condition corresponding to the target

$$
\begin{gathered}
\partial_{t}\left|\lambda_{l}(t)\right\rangle=-(i / \hbar)\left[H_{0}-\mu_{Z} \varepsilon(t)\right]\left|\lambda_{l}(t)\right\rangle \text { with } \\
\left|\lambda_{l}(t=T)\right\rangle=\left|l_{f}\right\rangle .
\end{gathered}
$$

Finally, variation of the field gives

$$
\begin{aligned}
\delta_{\varepsilon} J_{F}= & -2 \int_{0}^{T} \alpha(t) \varepsilon(t) \delta \varepsilon(t) d t \\
& -2 \Re e(-i / \hbar)\left[\sum_{j=1}^{2^{n}}\left\langle j_{f} \mid \psi_{j}(T)\right\rangle\right. \\
& \left.\times \sum_{k=1}^{2^{n}} \int_{0}^{T}\left\langle\lambda_{k}(t)\left|\mu_{Z} \delta \varepsilon(t)\right| \psi_{k}(t)\right\rangle d t\right] .
\end{aligned}
$$


To obtain $\delta_{\varepsilon} J_{F}$, the optimal field is

$$
\begin{aligned}
\varepsilon_{F}(t)= & -\frac{1}{\alpha(t)} \Im m \\
& \left(\sum_{j=1}^{2^{n}}\left\langle j_{f} \mid \psi_{j}(T)\right\rangle \sum_{k=1}^{2^{n}}\left\langle\lambda_{k}(t)\left|\mu_{Z}\right| \psi_{k}(t)\right\rangle\right) .
\end{aligned}
$$

By Eqs. (A3) and (A6), we use the time invariance of the overlap integrals $\left\langle j_{f} \mid \psi_{j}(T)\right\rangle$ and insert the time-dependent functions $\psi_{j}(t)$ and $\lambda_{j}(t)$ so that the field takes the form

$$
\begin{aligned}
\varepsilon_{F}(t)= & -\frac{1}{\alpha(t)} \Im m \\
& \left(\sum_{j=1}^{2^{n}}\left\langle\lambda_{j}(t) \mid \psi_{j}(t)\right\rangle \sum_{k=1}^{2^{n}}\left\langle\lambda_{k}(t)\left|\mu_{Z}\right| \psi_{k}(t)\right\rangle\right) .
\end{aligned}
$$

Note that one could use another functional by generalizing the one proposed in Ref. 62 in the single target case

$$
\begin{aligned}
J_{F}^{(2)}= & \left|\sum_{k=1}^{2^{n}}\left\langle k_{f} \mid \psi_{k}(T)\right\rangle\right|^{2} \\
& -\int_{0}^{T} \alpha(t) \varepsilon^{2}(t) d t-2 \Re e\left[\sum_{k=1}^{2^{n}} \int_{0}^{T}\left\langle\lambda_{k}(t)\right| \frac{\partial}{\partial t}\right. \\
& \left.+i\left[H_{0}-\mu_{Z} \varepsilon(t)\right]\left|\psi_{k}(t)\right\rangle d t\right] .
\end{aligned}
$$

Then variation of $\lambda_{l}$ still leads to the same Eq. (A3) with an initial condition $\left|\psi_{l}(t=0)\right\rangle=|l\rangle$ but variation of $\psi_{l}$ gives an equation with a different final condition $\left|\lambda_{l}(T)\right\rangle$ $=\sum_{j}^{2^{n}}\left\langle j_{f} \mid \psi_{j}(T)\right\rangle\left|l_{f}\right\rangle$ which is the target with a phase factor. The optimal field then takes the form

$$
\varepsilon_{F}^{(2)}(t)=-\frac{1}{\alpha(t)} \Im m\left[\sum_{k=1}^{2^{n}}\left\langle\lambda_{k}(t)\left|\mu_{Z}\right| \psi_{k}(t)\right\rangle\right] .
$$

A similar result has also been obtained by optimizing the real part of $\sum_{k=1}^{2^{n}}\left\langle k_{f} \mid \psi_{k}(T)\right\rangle .^{37,56,73}$

${ }^{1}$ C. H. Bennett, Int. J. Theor. Phys. 21, 905 (1982).

${ }^{2}$ M. A. Nielsen and I. L. Chuang, Quantum Computation and Quantum Information (Cambridge University Press, 2000).

${ }^{3}$ S. L. Braunstein and P. van Loock, Rev. Mod. Phys. 77, 513 (2005).

${ }^{4}$ J. I. Cirac and P. Zoller, Phys. Rev. Lett. 74, 4091 (1995).

${ }^{5}$ L.-M. Duan and C. Monroe, Rev. Mod. Phys. 82, 1209 (2010).

${ }^{6}$ R. Hanson, L. P. Kouwenhoven, J. R. Petta, S. Tarucha, and L. M. K. Vandersypen, Rev. Mod. Phys. 79, 1217 (2007).

${ }^{7}$ O. Morsch and M. Oberthaler, Rev. Mod. Phys. 78, 179 (2006).

${ }^{8}$ D. DeMille, Phys. Rev. Lett. 88, 067901 (2002).

${ }^{9}$ L. D. Carr, D. DeMille, R. V. Krems, and J. Ye, New J. Phys. 11, 055049 (2009).

${ }^{10}$ T. D. Ladd, F. Jelezko, R. Laflamme, Y. Nakamura, C. Monroe, and J. L. O'Brien, Nature (London) 464, 45 (2010).

${ }^{11}$ N. A. Gershenfeld and I. L. Chuang, Science 275, 350 (1997).

${ }^{12}$ C. M. Tesch, L. Kurtz, and R. de Vivie-Riedle, Chem. Phys. Lett. 343, 633 (2001).

${ }^{13}$ C. M. Tesch and R. de Vivie-Riedle, Phys. Rev. Lett. 89, 157901 (2002).

${ }^{14}$ J. Vala, Z. Amitay, B. Zhang, S. Leone, and R. Kosloff, Phys. Rev. A 66, 62316 (2002).

${ }^{15}$ Z. Amitay, R. Kosloff, and S. R. Leone, Chem. Phys. Lett. 359, 8 (2002).

${ }^{16}$ U. Troppmann, C. M. Tesch, and R. de Vivie-Riedle, Chem. Phys. Lett. 378, 273 (2003).

${ }^{17}$ C. M. Tesch and R. de Vivie-Riedle, J. Chem. Phys. 121, 12158 (2004).

${ }^{18}$ D. Babikov, J. Chem. Phys. 121, 7577 (2004).

${ }^{19}$ B. Korff, U. Troppmann, K. Kompa, and R. de Vivie-Riedle, J. Chem. Phys. 123, 244509 (2005).
${ }^{20}$ U. Troppmann and R. de Vivie-Riedle, J. Chem. Phys. 122, 154105 (2005).

${ }^{21}$ U. Troppmann, C. Gollub, and R. de Vivie-Riedle, New J. Phys. 8, 100 (2006).

${ }^{22}$ Y. Ohtsuki, Chem. Phys. Lett. 404, 126 (2005).

${ }^{23}$ T. Cheng and A. Brown, J. Chem. Phys. 124, 034111 (2006).

${ }^{24}$ M. Zhao and D. Babikov, J. Chem. Phys. 125, 024105 (2006).

${ }^{25}$ D. Sugny, C. Kontz, M. Ndong, Y. Justum, G. Dive, and M. DesouterLecomte, Phys. Rev. A 74, 043419 (2006).

${ }^{26}$ M. Ndong, L. Bomble, D. Sugny, Y. Justum, and M. Desouter-Lecomte, Phys. Rev. A 76, 043424 (2007).

${ }^{27}$ M. Ndong, D. Lauvergnat, X. Chapuisat, and M. Desouter-Lecomte, J. Chem. Phys. 126, 244505 (2007).

${ }^{28}$ D. Weidinger and M. Gruebele, Mol. Phys. 105, 1999 (2007).

${ }^{29}$ M. Zhao and D. Babikov, J. Chem. Phys. 126, 204102 (2007).

${ }^{30}$ M. Tsubouchi and T. Momose, Phys. Rev. A 77, 052326 (2008).

${ }^{31}$ L. Bomble, D. Lauvergnat, F. Remacle, and M. Desouter-Lecomte, J. Chem. Phys. 128, 064110 (2008).

${ }^{32}$ Y. Y. Gu and D. Babikov, J. Chem. Phys. 131, 034306 (2009).

${ }^{33}$ R. R. Zaari and A. Brown, J. Chem. Phys. 132, 014307 (2010).

${ }^{34}$ D. Sugny, L. Bomble, T. Ribeyre, O. Dulieu, and M. Desouter-Lecomte, Phys. Rev. A 80, 042325 (2009).

${ }^{35}$ L. Bomble, D. Lauvergnat, F. Remacle, and M. Desouter-Lecomte, Phys. Rev. A 80, 022332 (2009).

${ }^{36}$ L. Bomble, D. Lauvergnat, F. Remacle, and M. Desouter-Lecomte, Phys. Chem. Chem. Phys. 12, 15628 (2010).

${ }^{37}$ Y. Ohtsuki, New J. Phys. 12, 045002 (2010).

${ }^{38}$ K. Mishima and K. Yamashita, Chem. Phys. 367, 63 (2010).

${ }^{39}$ R. R. Zaari and A. Brown, J. Chem. Phys. 135, 044317 (2011).

${ }^{40}$ S. Sharma and H. Singh, Chem. Phys. 390, 68 (2011).

${ }^{41}$ S. F. Yelin, K. Kirby, and R. Côté, Phys. Rev. A 74, 050301 (2006).

${ }^{42}$ E. Kuznetsova, R. Côté, K. Kirby, and S. F. Yelin, Phys. Rev. A 78, 012313 (2008).

${ }^{43}$ E. Charron, P. Milman, A. Keller, and O. Atabek, Phys. Rev. A 75, 033414 (2007).

${ }^{44}$ L. Bomble, P. Pellegrini, P. Ghesquière, and M. Desouter-Lecomte, Phys. Rev. A 82, 062323 (2010).

${ }^{45}$ K. Mishima and K. Yamashita, Chem. Phys. 361, 106 (2009).

${ }^{46}$ K. Mishima and K. Yamashita, J. Chem. Phys. 130, 034108 (2009).

${ }^{47}$ Q. Wei, S. Kais, B. Friedrich, and D. Herschbach, J. Chem. Phys. 135, 154102 (2011)

${ }^{48}$ J. Zhu, S. Kais, Q. Wei, D. Herschbach, and B. Friedrich, J. Chem. Phys. 138, 024104 (2013).

${ }^{49}$ H. Ran, J. Aldegunde, and J. M. Hutson, New J. Phys. 12, 043015 (2010).

${ }^{50}$ S. Ospelkaus, K.-K. Ni, G. Quéméner, B. Neyenhuis, D. Wang, M. H. G. de Miranda, J. L. Bohn, J. Ye, and D. S. Lin, Phys. Rev. Lett. 104, 030402 (2010).

${ }^{51}$ B. L. Lev, E. R. Meyer, E. R. Hutson, B. C. Sawyer, J. L. Bohn, and J. Ye, Phys. Rev. A 74, 061402 (2006).

${ }^{52}$ E. R. Hudson, H. J. Lewandowski, B. C. Sawyer, and J. Ye, Phys. Rev. Lett. 96, 143004 (2006)

${ }^{53}$ P. Pellegrini and M. Desouter-Lecomte, Eur. Phys. J. D 64, 163 (2011).

${ }^{54}$ P. Pellegrini, S. Vranckx, and M. Desouter-Lecomte, Phys. Chem. Chem. Phys. 13, 18864 (2011).

${ }^{55}$ D. Shyshlov and D. Babikov, J. Chem. Phys. 137, 194318 (2012).

${ }^{56}$ J. P. Palao and R. Kosloff, Phys. Rev. Lett. 89, 188301 (2002).

${ }^{57}$ J. P. Palao and R. Kosloff, Phys. Rev. A 68, 062308 (2003).

${ }^{58}$ L. Wang and D. Babikov, J. Chem. Phys. 137, 064301 (2012).

${ }^{59}$ J. Aldegunde, B. A. Rivington, P. S. Zuchowski, and J. M. Hutson, Phys. Rev. A 78, 033434 (2008).

${ }^{60}$ J. Aldegunde, H. Ran, and J. M. Hutson, Phys. Rev. A 80, 043410 (2009).

${ }^{61}$ W. Zhu, J. Botina, and H. Rabitz, J. Chem. Phys. 108, 1953 (1998).

${ }^{62}$ W. Zhu and H. Rabitz, J. Chem. Phys. 109, 385 (1998).

${ }^{63}$ Y. Ohtsuki, G. Turinici, and H. Rabitz, J. Chem. Phys. 120, 5509 (2004).

${ }^{64}$ K. Sundermann and R. de Vivie-Riedle, J. Chem. Phys. 110, 1896 (1999).

${ }^{65}$ M. Hayashi, Quantum Information (Springer, Berlin, 2006)

${ }^{66}$ A. Gilchrist, N. K. Langford, and M. A. Nielsen, Phys. Rev. A 71, 062310 (2005).

${ }^{67}$ X. Wang and S. G. Schirmer, Phys. Rev. A 79, 052326 (2009). 
${ }^{68}$ M. A. Nielsen, Phys. Lett. A 303, 249 (2002).

${ }^{69}$ M. D. Bowdrey, D. K. L. Oi, A. J. Short, K. Banaszek, and J. A. Jones, Phys. Lett. A 294, 258 (2002).

${ }^{70}$ J. F. Poyatos, J. I. Cirac, and P. Zoller, Phys. Rev. Lett. 78, 390 (1997).
${ }^{71}$ W. H. Press, S. A. Teukolsky, W. T. Vetterling, and B. P. Flannery, Numerical Recipes in FORTRAN: The Art of Scientific Computing (Cambridge University Press, Cambridge, 1986).

${ }^{72}$ M. Sugawara and Y. J. Fujimura, J. Chem. Phys. 100, 5646 (1994).

${ }^{73}$ M. H. Goerz, T. Calareo, and C. P. Koch, J. Phys. B 44, 154011 (2011). 